\title{
Badis singenensis, a new fish species (Teleostei: Badidae) from Singen River, Arunachal Pradesh, northeastern India
}

\section{K. Geetakumari ${ }^{1}$ \& Kento Kadu ${ }^{2}$}

${ }^{1}$ Department of Life Sciences, Manipur University, Canchipur, Imphal, Manipur 795003, India

Present addresss: ICAR Research Complex for NEH Region, Manipur Centre, Lamphelpat, Imphal, Manipur 795004, India

${ }^{2}$ Department of Zoology, Jawaharlal Nehru College, Pasighat, East Siang District, Arunachal Pradesh 791102, India

Email: ${ }^{1}$ geetameme@gmail.com (corresponding author), ${ }^{2}$ kentokadu@yahoo.com

\begin{abstract}
Anew species of Badis from Singen River, Brahmaputra basin in Arunachal Pradesh, India, has the following combination of characters: a conspicuous round black blotch postero-dorsally on opercle at the base of opercle spine covering many scales: three distinct black blotches at dorsal fin base: the first, behin the third spine; the second, behind the sixth dorsal spine and the third, behind the fifth and sixth soft dorsal rays. The species differs from its nearest congeners, $B$. assamensis and $B$. blosyrus by the presence of a black blotch at the base behind the fifth soft anal fin ray.
\end{abstract}

Keywords: Arunachal Pradesh, Brahmaputra basin, new fish, Perciformes.

The Indo-Burmese genus Badis Bleeker is characteristic in having an opercle with a single sharp spine at its postero-dorsal corner; contiguous spinous and soft dorsal fins; the base of the soft part longer than that of the spinous part; anal fin with three

Date of publication (online): 26 September 2011

Date of publication (print): 26 September 2011

ISSN 0974-7907 (online) | 0974-7893 (print)

Editor: K. Rema Devi

\section{Manuscript details:}

Ms \# 02531

Received 02 August 2010

Final received 06 June 2011

Finally accepted 12 August 2011

Citation: Geetakumari, K. \& K. Kadu (2011). Badis singenensis, a new fish species (Teleostei: Badidae) from Singen River, Arunachal Pradesh, northeastern India. Journal of Threatened Taxa 3(9): 2085-2089.

Copyright: () K. Geetakumari \& Kento Kadu 2011. Creative Commons Attribution 3.0 Unported License. JoTT allows unrestricted use of this article in any medium for non-profit purposes, reproduction and distribution by providing adequate credit to the authors and the source of publication.

Acknowledgements: The authors are grateful to Prof. W. Vishwanath, Department of Life Sciences, Manipur University for his valuable suggestions; to Dr. D.N. Das, Department of Zoology, Rajiv Gandh University, Itanagar for his valuable help and to Dr. Kenjum Bagra, Research Officer, Arunachal Pradesh, Biodiversity Board, Itanagar for his precious information. The first author records her thankfulness to Department of Biotechnology for financial assistance for DBT-RA programme.

OPEN ACCESS I FREE DOWNLOAD (C) (i) (C) spines; lateral line pores tubed and interrupted; jaws, vomer and palatines with villiform teeth; scales both ctenoid and cycloid; 2-4 dentary foramina; 3-toothed hypobranchial; short pelvic fin in males, not reaching the first dorsal spine; short dorsal fin lappets and rounded caudal fin (Kullander \& Britz 2002).

As many as 14 species of Badis are currently treated valid, of which six are from Brahmaputra drainage-Badis assamensis Ahl, B. badis (Hamilton), B. blosyrus Kullander \& Britz, B. dibruensis Geetakumari \& Vishwanath, B. kanabos Kullander \& Britz, and B. tuivaiei Vishwanath \& Shanta; five from Irrawaddy drainage - B. corycaeus Kullander \& Britz, B. ferrarisi Kullander \& Britz, B. kyar Kullander \& Britz, B. pyema Kullander \& Britz, and B. ruber Schreitmiiller and one each from Matamohuri River drainage, of Takaupa River basin and Mae Nam Khwae Noi drainage, respectively, B. chittagongis Kullander and Britz, B. siamensis Klausewitz and B. khwae Kullander and Britz.

During field surveys in northeastern India during 2008-2009, 27 specimens of an undescribed Badis were collected from Singen River, Brahmaputra basin, Arunachal Pradesh (Fig. 1). The species is herein described as Badis singenensis sp. nov.

\section{Materials and Methods}

Measurements were made with dial calipers to the nearest $0.1 \mathrm{~mm}$ and expressed as percentages of standard length (SL). Counts and measurements were made on the left side of specimens under a PC-based binocular stereozoom microscope (Olympus SZ40) with transmitted light. Counts and measurements followed Kullander \& Britz (2002), clearing and staining of specimens for osteology after Hollister (1934) and identification and nomenclature of bones 


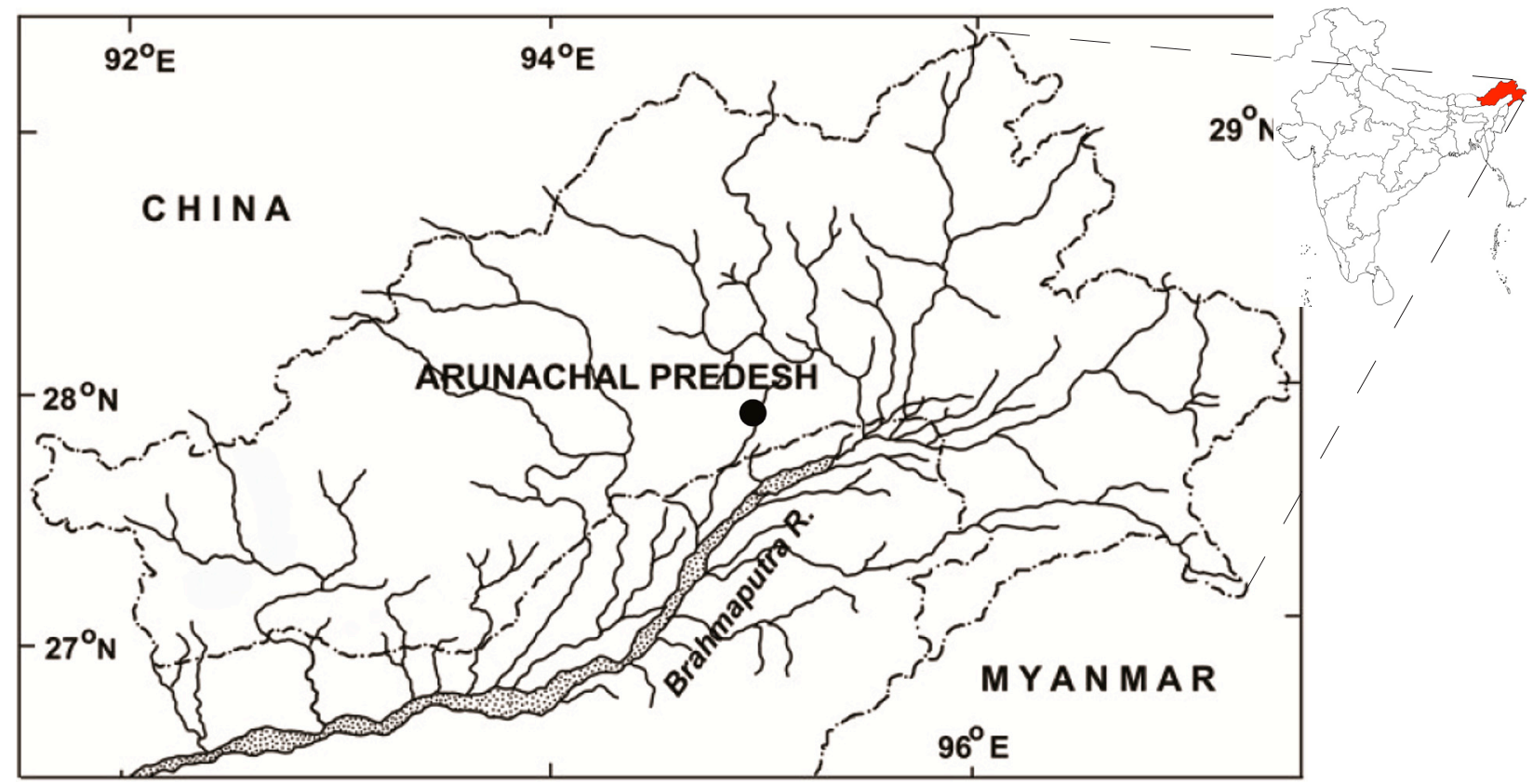

Figure 1. Map showing distribution of Badis singenensis sp. nov.

and vertebral counts after Greenwood (1976). For branchial toothplate count, the first gill arch on the left side of the specimens was taken and plates starting from hypobranchial to epibranchial of the outer side were counted. Type specimens are deposited in the Manipur University Museum of fishes (MUMF) and Rajiv Gandhi University Museum of Fishery (RGUMF)

\section{Type material}

Badis singenensis sp. nov. (Image 1a-c)

Holotype: 25.ii.2008, 22.3mm SL, 27054'18.72”N \& 94'55'21.12"E, Brahmaputra drainage, Singen River, Saku-Kadu Village, Arunachal Pradesh, India, coll. Rikge Kadu \& Kento Kadu (MUMF-Per 112).

Paratypes: 26 exs., RGUMF 0218-0225, 27.0$37.0 \mathrm{~mm} \mathrm{SL}$, same data as of holotype; MUMF-Per 113-131, 19, 24.4-42.0 mm SL, data as for holotype; MUMF Per-119-120, 2, dissected, cleared and stained for osteology.

\section{Diagnosis}

The new Badis singenensis sp. nov. is with the following combination of characters: a conspicuous black blotch posterodorsally on opercle, at the base of opercle spine, round and usually covering portion
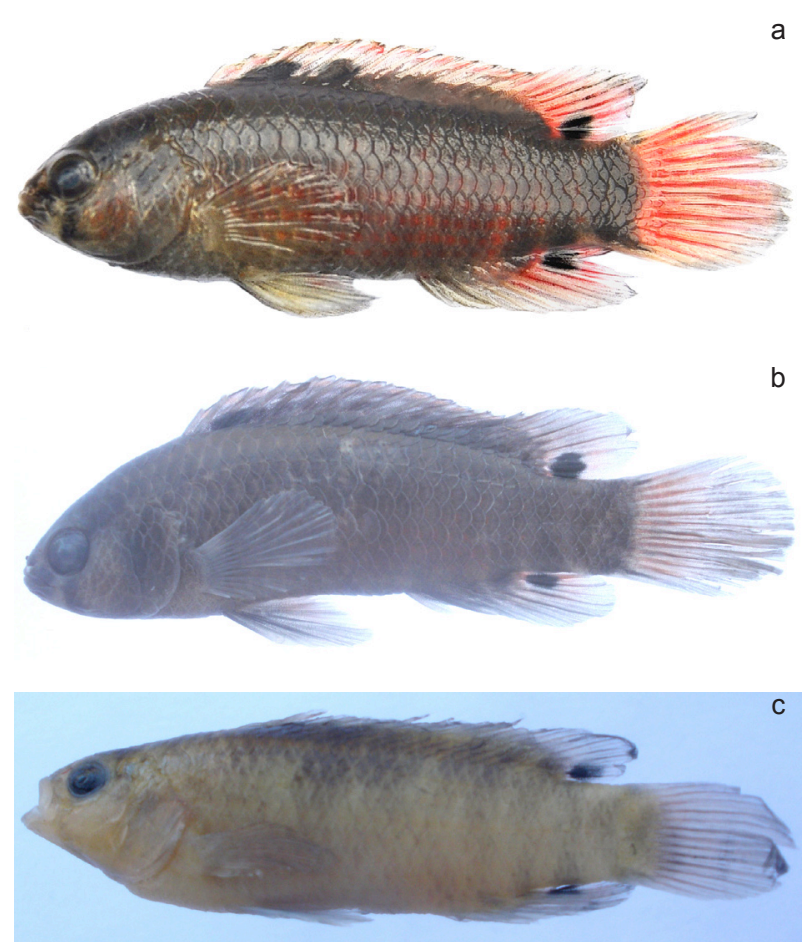

Image 1. a - Side view of Badis singenensis sp. nov. (uncat.) showing colouration; b - (RGUMF- 0218, paratype, female); c - (RGUMF- 0219, paratype, male) 
of several scales; three distinct dark blotches at dorsal fin base, first blotch behind third spine, second behind sixth dorsal spine and third behind the fifth and sixth soft dorsal ray; another distinct black blotch at the base of anal fin behind the fifth soft anal fin ray; tooth plate count 5; scales in lateral row 25-26; interorbital width 9.2-13.3\% SL; upper jaw length 7.6-8.8\% SL; lower jaw length $9.4-10.2 \%$ SL; head length $30.2-34.6 \%$ SL.

\section{Description}

Morphometric data and counts are in Tables $1 \& 2$, respectively. Frequency distributions of meristic characters are in Table 3 and comparison with related species, in Table 4. Body elongate, moderately compressed laterally. Predorsal profile in small specimens straight, sloping at some angle similar prepelvic profile in larger specimens and more strongly as the size increases. Head pointed in lateral aspect. Orbit situated in anterior half of head at about mid-lateral axis of body and moderately large, diameter about one-third of head length. Mouth oblique and moderately large, protrusible, lower jaw slightly projecting beyond upper, maxilla reaching to $1 / 3$ of orbit. Opercular spine slender, with a sharp tip. Palatine, vomer and parasphenoid toothed.

Pores: dental three, anguloarticular two, preopercular six, nasal three, supraorbital three, extrascapular two, supracleithral two, posttemporal two, coronalis one, lachrymal three, infraorbital pores three. A row of free neuromasts extending across the gap between lachrymal and anteriormost infraorbital.

Scales strongly ctenoid on sides, cycloid on top of head. On the ventral side, the sizes of the scales become reduced towards the posterior side. Predorsal scales anterior to coronalis pore 4-5, posteriorly 8-9. Cheek and opercular scales ctenoid, 4-5 rows of scales on cheek. Three rows of scales on opercle, one row each on preopercle, subopercle and interopercle, a few scales anterior to cheek cycloid. Lateral line divided into two segments, with anterior segment more dorsally located than posterior segment. Upper lateral line begins at dorsal origin of operculum. Lower lateral line begins at vertical through the posterior end of anal fin origin, vertically centered along length of caudal peduncle. Circumpeduncular scale rows nine above, nine below lateral line, totaling 19. Dorsal fin scale cover up to 3-4 scales wide; anal fin scale cover three
Table 1. Proportional measurements of Badis singenensis sp. nov. in percentage of standard length except standard length.

\begin{tabular}{|l|c|c|c|c|c|}
\hline & \multirow{2}{*}{ Holotype } & \multicolumn{4}{|c|}{ Paratypes N=26 } \\
\cline { 3 - 6 } & & Mean & Min. & Max. & S.D. \\
\hline Standard length $(\mathrm{mm})$ & 0.85 & & & & \\
\hline Head length & 32.9 & 32.0 & 30.2 & 34.6 & 1.6 \\
\hline Snout length & 7.1 & 7.8 & 6.9 & 9.2 & 0.9 \\
\hline Orbital diameter & 12.9 & 10.4 & 8.1 & 12.9 & 1.9 \\
\hline Interorbital width & 9.4 & 10.7 & 9.2 & 13.3 & 1.5 \\
\hline Upper jaw length & 8.2 & 8.3 & 7.6 & 8.8 & 0.4 \\
\hline Lower jaw length & 9.4 & 9.8 & 9.4 & 10.2 & 0.4 \\
\hline Body depth & 29.4 & 30.2 & 29.4 & 30.6 & 0.5 \\
\hline Pelvic fin length & 25.9 & 24.7 & 23.4 & 25.9 & 0.9 \\
\hline Pelvic to anal fin distance & 29.4 & 30.9 & 27.6 & 37.2 & 3.3 \\
\hline
\end{tabular}

Table 2. Counts of Badis singenensis sp. nov.

\begin{tabular}{|l|c|c|c|}
\hline \multirow{2}{*}{ Counts } & \multirow{2}{*}{ Holotype } & \multicolumn{2}{|c|}{ Paratypes } \\
\cline { 3 - 4 } & & Min. & Max. \\
\hline D & $15 / 7$ & $15 / 7$ & $15 / 8$ \\
\hline P & 14 & 14 & 14 \\
\hline A & iii,6 & 6 & 7 \\
\hline Lateral scale rows & 32 & 29 & 32 \\
\hline Lateral line count & $21 / 5$ & $21 / 4$ & $21 / 5$ \\
\hline Lateral transverse scales & $11 / 2 / 1 / 7$ & $11 / 2 / 1 / 7$ & $11 / 2 / 1 / 7$ \\
\hline Circumpeduncular scales & 19 & 19 & 20 \\
\hline Toothplate count & & 5 & 5 \\
\hline Vertebrae & & 28 & 28 \\
\hline
\end{tabular}

scales wide. Scales in vertical row $1 \frac{1}{2}$ above, seven below lateral lines. Toothplates on the first branchial arch five, vertebra 28 (16/12).

Dorsal fin with long base, anterior insertion vertically above the pectoral fin insertion and posterior insertion at vertical through base of last anal-fin ray. Soft dorsal and anal fins with rounded tips reaching to almost about $1 / 2$ or $1 / 3$ of caudal fin. Caudal fin rounded. Pectoral fin rounded, extending about $2 / 3$ distance to anal- fin origin. Pelvic fin elongated and pointed, inner branch of second soft ray longest, not reaching up to vent, but terminating close to vent in some large specimens. Head length, orbital diameter, interorbital width, upper jaw length and lower jaw length respectively $(30.2-34.6),(8.1-12.9)$, (9.2$13.3),(7.6-8.8)$ and $(9.4-10.2) \%$ SL. 
Table 3. Frequency distribution of meristic characters

\begin{tabular}{|c|c|c|c|c|c|c|}
\hline$a$ & $\begin{array}{l}\text { Dorsal fin counts (spines/soft rays) } \\
\text { Specimens }\end{array}$ & $\begin{array}{c}15 / 8 \\
1\end{array}$ & $\begin{array}{c}15 / 7 \\
8\end{array}$ & $\begin{array}{c}16 / 9 \\
3\end{array}$ & $\begin{array}{c}17 / 8 \\
3\end{array}$ & \\
\hline b & $\begin{array}{l}\text { Anal fin counts } \\
\text { Specimens }\end{array}$ & $\begin{array}{l}5 \\
2\end{array}$ & $\begin{array}{l}6 \\
7\end{array}$ & $\begin{array}{l}7 \\
3\end{array}$ & $\begin{array}{l}8 \\
3\end{array}$ & \\
\hline c & $\begin{array}{l}\text { Pectoral fin counts } \\
\text { Specimens }\end{array}$ & $\begin{array}{c}13 \\
4\end{array}$ & $\begin{array}{l}14 \\
11\end{array}$ & & & \\
\hline$d$ & $\begin{array}{l}\text { Lateral scale rows } \\
\text { Specimens }\end{array}$ & $\begin{array}{c}29 \\
1\end{array}$ & $\begin{array}{c}30 \\
3\end{array}$ & $\begin{array}{c}31 \\
9\end{array}$ & $\begin{array}{c}32 \\
-\end{array}$ & $\begin{array}{c}33 \\
2\end{array}$ \\
\hline $\mathrm{e}$ & $\begin{array}{l}\text { Lateral line scale counts (upper/lower } \\
\text { scales) } \\
\text { Specimens }\end{array}$ & $\begin{array}{c}20 / 5 \\
1\end{array}$ & $\begin{array}{c}21 / 4 \\
1\end{array}$ & $\begin{array}{c}20 / 6 \\
1\end{array}$ & $\begin{array}{c}21 / 5 \\
10\end{array}$ & $\begin{array}{c}22 / 4 \\
2\end{array}$ \\
\hline$f$ & $\begin{array}{l}\text { Tooth plate } \\
\text { Specimens }\end{array}$ & $\begin{array}{l}5 \\
3\end{array}$ & $\begin{array}{l}6 \\
1\end{array}$ & & & \\
\hline $\mathrm{g}$ & $\begin{array}{l}\text { Vertebrae number } \\
\text { Specimens }\end{array}$ & $\begin{array}{c}15 / 13 \\
2\end{array}$ & $\begin{array}{c}15 / 14 \\
1\end{array}$ & & & \\
\hline
\end{tabular}

Table 4. Comparison of proportional measurements in \% SL and counts of Badis singenensis sp. nov. with related species.

\begin{tabular}{|l|c|c|c|}
\hline Proportions & B. singenensis sp.nov. & B. assamensis & B. blosyrus \\
\hline Tooth plate count & 5 & $7-9$ & $10-13$ \\
\hline Interorbital width & $11.25(9.2-13.3)$ & $5.4(4.8-6.0)$ & $7.4(6.4-8.0)$ \\
\hline Upper jaw length & $8.2(7.6-8.8)$ & $10.3(9.7-10.9)$ & $12.8(12.0-13.6)$ \\
\hline Lower jaw length & $9.8(9.4-10.2)$ & $13.7(12.7-14.6)$ & $17.4(16.3-18.5)$ \\
\hline Head length & $32.4(30.2-34.6)$ & $31.9(29.2-34.5)$ & $37.4(36.0-38.8)$ \\
\hline Scales in lateral row & $26(25-26)$ & $28-29$ & $27(27-28)$ \\
\hline
\end{tabular}

\section{Colouration}

Live colours: body dark slaty gray dorsally, paler on sides, each scale with reddish tinge; dorsal fin reddish, tips of fin rays black. Caudal fin orange, paired fins and anal fin slaty gray with reddish tinge. Posterodorsally on opercle, at base of opercular spine, a rounded black spot covering 3-4 scales. Three distinct dark black blotches surrounded by red coloration at dorsal fin base: the first behind the third dorsal spine, the second behind the sixth dorsal spine and the third, behind the fifth dorsal soft ray. A black blotch at the base behind the fifth soft anal fin ray.

In 10\% formalin: supraorbital stripe prominent, rounded black opercular spot prominent. Body with 5-6 broad black bars. Black bar on caudal peduncle well separated from the preceeding bar between posterior ends of dorsal and anal fin bases. Black blotches on dorsal fin base and soft anal fins prominent. Dorsal fin gray with narrow, contrasting white margin, and each lappet with a blackish submarginal stripe.

\section{Sexual dimorphism}

Males have brighter body colour than their female counterpart. In the male vertical bars on posterior portion of lateral body are more distinct than on the female which are less distinct or absent. During the breeding season (April to June) males develop a red coloured mark on their soft dorsal and soft anal fin and in some female specimens red coloured marks were observed in lateral scales. Females have a deeper body height than the males.

\section{Etymology}

The species is named after the Singen River, Arunachal Pradesh, type locality of the species.

\section{Distribution}

Presently known only from Singen River at SakuKadu Village, East Siang District, Arunachal Pradesh, Brahmaputra drainage, northeastern India.

\section{Discussion}

Badis singenensis is distinguished from all its congeners by the presence (vs. absence) of black blotches on the dorsal fin and one on the soft anal fin. It further differs from its nearest congener, B. assamensis 
in having fewer tooth plates (5 vs. 7-9), scales in lateral row (25-26 vs. 28-29); wider interorbital region (9.2-13.3 vs. $4.8-6.0 \% \mathrm{SL})$; shorter upper jaw (7.6-8.8 vs.9.7-10.9\% SL) and lower jaw (9.4-10.2 vs. $12.7-14.6 \% \mathrm{SL}$ ). It is also distinguished from $B$. blosyrus in having fewer tooth plates (5 vs. 10-13); less scales in lateral row (25-25 vs. 27-28); shorter head length (30.2-34.6 vs. $36.0-38.8 \%$ SL); wider interorbital space (9.2-13.3 vs. $6.4-8.0 \% \mathrm{SL})$; shorter upper jaw (7.6-8.8 vs.12.0-13.6\%SL) and lower jaw (9.4-10.2 vs. $16.3-18.5 \%$ SL).

Badis singenensis differs from its congeners from the Brahmaputra basin, viz., B. badis, $B$. dibruensis, $B$. kanabos and B. tuivaiei by the presence (vs. absence) of black blotch on the soft anal fin. It further differs from $B$. badis in having wider interorbital space (9.213.3 vs. $6.5-8.3 \% \mathrm{SL})$ and shorter lower jaw (9.4-10.2 vs. $11.3-14.5 \% \mathrm{SL}$ ). It also differs from $B$. dibruensis in having longer upper jaw (7.6-8.8 vs. $6.1-6.9 \% \mathrm{SL}$ ) and lower jaw (9.4-10.2 vs. $7.8-8.3 \%$ SL). It further differs from B. kanabos in having wider interorbital space $(9.2-13.3$ vs. $7.3-8.6 \% \mathrm{SL})$ and shorter lower jaw (9.4-10.2 vs. $11.0-13.5 \% \mathrm{SL})$. It also further differs from $B$. tuivaiei in having wider interorbital space (9.2-13.3 vs. 5.6-7.2\% SL) and shorter lower jaw (9.4-10.2 vs 10.9-16.4).

The new species differs from all the five species of the Irrawaddy drainage in the presence (vs. absence) of opercle blotch and in the presence (vs. absence) of black blotch on the soft dorsal fin. It further differs from $B$. ferrarisi in having longer interorbital width (9.2-13.3\% SL) and longer lower jaw (11.3-12.8 vs. 9.4-10.2\% SL).

Badis singenensis also differs from $B$. siamensis and $B$. khwae in the absence (vs. presence) of opercle blotch and from B. chittagongis in absence (vs. presence) of opercle blotch.

Kullander \& Britz (2002) classified the species of Badis into five groups viz., B. ruber, B. assamensis, $B$. corycaeus, B. kyar and B. badis group. They reported $B$. assamensis group to be characteristic in having an opercle blotch and believed that more numbers of undescribed species of the group exist in the region. The new species under description belongs to the $B$. assamensis group.

\section{Comparative materials}

Badis assamensis: MUMF Per-51-54, 4, 41.6-55.8 mm SL; India; Assam, Dibrugarh, Dibru River; Badis assamensis: RGUMF-0180, 2, 49-52 mm SL, Dibang river, Lohit, Arunachal Pradesh, India; B. badis: MUMF Per-55-65, 11, 23.5-28.7 mm SL; India; Manipur, Barak River; Badis badis: RGUMF- 0149, 5, 23-40 mm SL, Mebang River, Arunachal Pradesh, India; B. blosyrus: MUMF Per-66-68, 3, 36.8-38.9 mm SL; India; Arunachal Pradesh, Lohit River; $B$. dibruensis: MUMF- Per 95, Holotype, 1, 37.3mm SL; India; Assam, Dibrugarh, Dibru River; B. ferrarisi: MUMF Per- 69-75, 7, 32.0-44.0 mm SL; India; Manipur, Lokchao River; B. kanabos: MUMF Per-7681, 6, 48.7-54.9 mm SL; India; Manipur, Barak River; B. tuivaiei: MUMF 5125-5132, 8, 53.5-59.4 mm SL; India; Manipur, Tuivai and Irang River.

\section{REFERENCES}

Greenwood, P.H. (1976). A review of the family centropomidae (Pisces, Perciformes). Bulletin of the British Museum (Natural History) 29(1): 1-81.

Hollister, G. (1934). Clearing and dyeing fish for bone study. Zoologica 12: 89-101.

Kullander, S.O. \& R. Britz (2002). Revision of the family Badidae (Teleostei: Perciformes), with description of a new genus and ten new species. Ichthyological Exploration of Freshwaters 13(4): 295-372. 